\title{
A novel algorithm for finding top-k weighted overlapping densest connected subgraphs in dual networks
}

\author{
Riccardo Dondi ${ }^{1}$, Mohammad Mehdi Hosseinzadeh ${ }^{1}$ and Pietro H. Guzzi ${ }^{2^{*}}$ (1)
}

\author{
${ }^{*}$ Correspondence: \\ hguzzi@unicz.it \\ ${ }^{2}$ Department of Surgical \\ and Medical Sciences, Magna \\ Graecia University, Catanzaro, \\ Italy \\ Full list of author information \\ is available at the end of the \\ article
}

\begin{abstract}
The use of networks for modelling and analysing relations among data is currently growing. Recently, the use of a single networks for capturing all the aspects of some complex scenarios has shown some limitations. Consequently, it has been proposed to use Dual Networks (DN), a pair of related networks, to analyse complex systems. The two graphs in a DN have the same set of vertices and different edge sets. Common subgraphs among these networks may convey some insights about the modelled scenarios. For instance, the detection of the Top-k Densest Connected subgraphs, i.e. a set k subgraphs having the largest density in the conceptual network which are also connected in the physical network, may reveal set of highly related nodes. After proposing a formalisation of the approach, we propose a heuristic to find a solution, since the problem is computationally hard. A set of experiments on synthetic and real networks is also presented to support our approach.
\end{abstract}

Keywords: Dual networks, Network mining, Dense subgraphs, Graph algorithms

\section{Introduction}

In last years, the use of networks to manage and analyse experimental data in many fields has grown (Cannataro et al. 2010; Barabási 2011). For instance, in computational biology associations among biological molecules (such as genes, proteins, small lipids etc.), are usually modelled as graphs. Data collected from social networks are modelled using graph theory and their analysis may shed light into association patterns among users (Sapountzi and Psannis 2018; Abatangelo et al. 2009; Clark and Kalita 2014; Faisal et al. 2015; Cannataro et al. 2010)

Usually, data are modelled using a single network whose nodes represent entities and edges their relations. Then, the topological analysis of the networks, i.e. global or local structures (Cannataro et al. 2010), finds context specific properties such as groups of related genes in biology or users in social networks (Liu et al. 2018). More recently, some works demonstrated that the use of a single network may not be able to capture all the relationships among elements considered, therefore some complex models have been introduced such as heterogeneous networks (Milano et al. 2020) or dual networks

(c) The Author(s) 2021. This article is licensed under a Creative Commons Attribution 4.0 International License, which permits use, sharing adaptation, distribution and reproduction in any medium or format, as long as you give appropriate credit to the original author(s) and the source, provide a link to the Creative Commons licence, and indicate if changes were made. The images or other third party material in this article are included in the article's Creative Commons licence, unless indicated otherwise in a credit line to the material. If material is not included in the article's Creative Commons licence and your intended use is not permitted by statutory regulation or exceeds the permitted use, you will need to obtain permission directly from the copyright holder. To view a copy of this licence, visit http://creativecommons. org/licenses/by/4.0/. 
(Wu et al. 2016). A dual network is a pair of related graphs sharing the same node set, with two different edge sets. One network has unweighted edges, and it is called physical graph. The second one has weighted edges and it is called conceptual graph. For example, in biology dual networks have been used to model interactions among genetic variants (Phillips 2008), where genetic interactions are modelled using the physical network and the quantitative effects of these interactions are modelled with the conceptual one.

An interesting problem in dual networks is the Densest Connected Subgraph (DCS) problem, that is finding a common subgraph between the two networks that has two properties: it is connected on the physical one and it is densest in the conceptual one. A DCS in a dual network may convey relevant information. For instance (Guzzi et al. 2020), showed that DCS may suggest missing links in social networks, capture similar interests among authors in a co-authorships dual network, where physical network represents co-authors and the conceptual network is used to model topics shared.

The relevance of problem arises in many real life scenarios. For instance in Phillips (2008) authors extracted a DCS from dual networks to analyse interactions between genetic variants and their strength. Given two input graphs $G_{c}\left(V, E_{c}\right)$ (undirected and edge-weighted), and $G_{p}\left(V, E_{p}\right)$ (undirected and unweighted), the problem consists in finding a subset of nodes $I_{s}$ that induces a densest community in $G_{c}$ and a connected subgraph in $G_{p}$. As proved in Wu et al. (2016), the DCS problem is NP-hard, since it may be reduced from the Set Cover problem (Karp 2009). Therefore there is the need for novel heuristics and computational approaches to solve it. Here we focus on a generalisation of this problem, since we search for a set of (overlapping) common subgraphs, that are connected in the physical network and densest in the conceptual network, i.e. top-k weighted overlapping densest connected subgraphs. The identification of top k-densest overlapping subgraphs in a network has been considered in Galbrun et al. (2016); Dondi et al. (2019); Hosseinzadeh (2020).

Our approach is based on a two step strategy: first a single alignment graph is built from the dual networks Guzzi and Milenković (2017); Milano et al. (2020), then we look for dense subgraphs in this network with an ad-hoc heuristic. Notice that these subgraphs correspond to dense subgraphs in the conceptual networks and connected subgraphs in the physical one, therefore they are solutions of the initial problem. Figure 1 depicts the workflow of our approach.

Considering the state of the art, we should note that we allow more flexibility with respect to other works such as Wu et al. (2016). In this work authors do not consider overlapping subgraphs and their approach is limited to the exact correspondence of nodes between networks. On the other hand, with respect to other approaches for finding densest subgraphs in a network (Balalau 2015; Galbrun et al. 2016; Dondi et al. 2019; Guzzi and Cannataro 2010), we consider weighted networks, an extension that can be useful in many contexts, in particular for biological and social networks.

We provide an implementation of our heuristic, and we show the effectiveness of our approach on synthetic datasets and on four real networks (a social network, two biological networks, a co-authorship network). The experimental results confirm the effectiveness of our approach.

The paper is structured as follows: "Related work" section discusses related works, "Definitions" section gives definitions and formally introduces the problem we are 


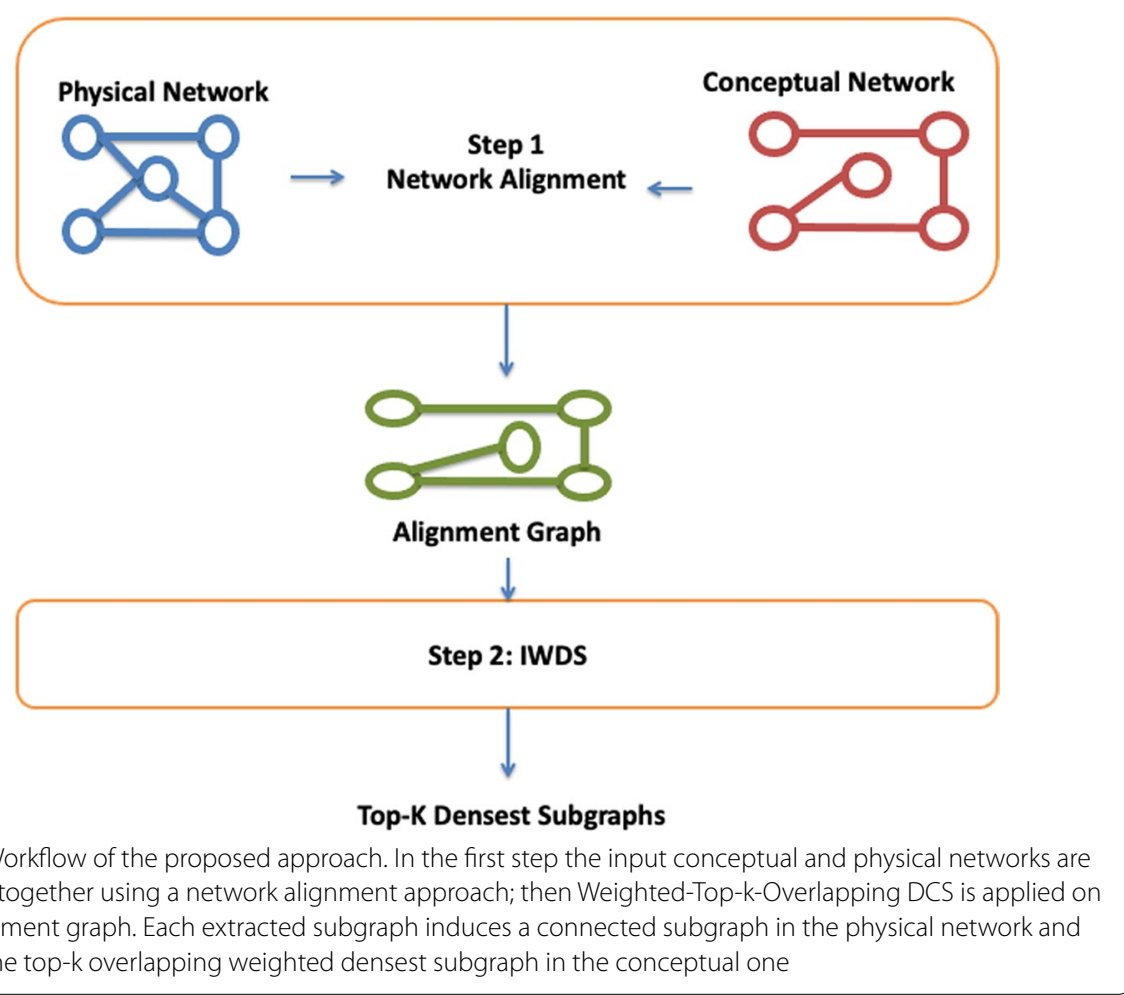

interested into. "The proposed algorithm" section presents our heuristic; "Experiments" section discusses the case studies; finally "Conclusion" section concludes the paper.

\section{Related work}

Many complex systems cannot be efficiently modelled using a single network without losses of information. Therefore the use of dual networks is growing (Wu et al. 2016; Sun and Kardia 2010). These applications span a large number of fields as introduced before: from bioinformatics to social networks. In genetics, dual networks are used to describe and analyse interactions among genetic variants. They can discover the common effects among multiple genetic variants (Sun and Kardia 2010), using a protein-protein interaction network that represents physical interactions and a weighted network that represents the relations between two genetic variants, usually measured by statistical tests.

A relevant problem in network analysis is that of discovering dense communities, as they represent strongly related nodes. The problem of finding communities in a network or a dual network is based on the specific model of dense or cohesive graph considered. Several models of cohesive subgraph have been considered in the literature and applied in different contexts. One of the first definition of a cohesive subgraph is a fully connected subgraph, i.e. a clique. However, the determination of a clique of the maximum size, also referred to as the Maximum Clique Problem, is NP-hard (Hastad 1996), and it is difficult to approximate (Zuckerman 2006). Moreover, in real networks communities may have missing edges; therefore, the clique model is often too strict and may fail to find some important subgraphs. Consequently, many alternative definitions of cohesive 
subgraphs that are not fully interconnected have been introduced, including $s$-club, $s$-plex and densest subgraph (Komusiewicz 2016; Dondi et al. 2019).

A densest subgraph is a subgraph with maximum density (where the density is the ratio between the number of edges and number of nodes of the subgraph) and the DensestSubgraph problem asks for a subgraph of maximum density in a given graph. The problem can be solved in polynomial time (Goldberg 1984; Kawase and Miyauchi 2018) and approximated within factor $\frac{1}{2}$ (Asahiro et al. 2000; Charikar 2000). Notice that the Densest-Subgraph problem can be extended also to edge-weighted networks.

Recently, Wu et al. (2016), proposed an algorithm for finding a densest connected subgraph in a dual network. The approach is based on a two-step strategy. In the first step, the algorithm prunes the dual network without eliminating the optimal solution. In the second step, two greedy approaches are developed to build a search strategy for finding a densest connected subgraph. Briefly, the first step finds the densest subgraph in the conceptual network. The second step refines this subgraph to guarantee that it is connected in the physical network.

In this contribution we use an approach based on local network alignment (LNA) that aims to find (relatively) small regions of similarity among two or more input networks. Such regions may be overlapping or not, and they represent conserved topological among networks. For instance, in protein interaction networks these regions are related to conserved motifs or pattern of interactions (Guzzi and Milenković 2017). LNA algorithms are usually based on building an intermediate structure, defined as alignment graph, and on the subsequent mining of it (Milano et al. 2020). For instance, Ciriello et al. (2012) and its successor AlignMCL (Mina and Guzzi 2014) are based on the construction of alignment graphs (see related papers for complete details about the construction of the alignment graph). GLAlign (Global Local Aligner), is a new local network alignment methodology (Milano et al. 2018) that mixes topology information from global alignment and biological information according to a linear combination schema, while the more recent L-HetNetAligner (Milano et al. 2020) extends the local alignment to heterogeneous networks.

While the literature of network mining has mainly focused on the problem of finding a single subgraph, recently the interest in finding more than a subgraph has emerged (Balalau 2015; Galbrun et al. 2016; Dondi et al. 2019; Hosseinzadeh 2020; Cho et al. 2013). The proposed approaches usually allows overlapping between the computed dense subgraphs. Indeed, there can be nodes that are shared between interesting dense subgraphs, for example hubs. The proposed approaches differ in the way they deal with overlapping. The problem defined in Balalau (2015) controls the overlap by limiting the Jaccard coefficient between each pair of subgraphs of the solution. The Top-k-Overlapping problem, introduced in Galbrun et al. (2016), includes a distance function in the the objective function. In this paper, we follow this last approach and we extend it to weighted networks.

\section{Definitions}

This section introduces the main concepts related to our problem.

Definition 1 Dual Network. 
A Dual Network (DN) $G\left(V, E_{c}, E_{p}\right)$ is a pair of networks: a conceptual weighted network $G_{c}\left(V, E_{c}\right)$ and a physical unweighted one $G_{p}\left(V, E_{p}\right)$.

Now, we introduce the definition of weighted density of a graph.

Definition 2 Density.

Given a weighted graph $G(V, E$, weight $)$, let $v \in V$ be a node of $G$, and let

$$
\operatorname{vol}(v)=\sum_{w:(v, w) \in E} \operatorname{weight}(v, w)
$$

be the sum of the weights of the edges incident in $v$. The density of the weighted graph $G$ is defined as

$$
\rho(G)=\frac{\sum_{v \in V} \operatorname{vol}(v)}{|V|} .
$$

Given a graph (weighted or unweighted) $G$ with a set $V$ of nodes and a subset $Z \subseteq V$, we denote by $G[Z]$ the subgraph of $G$ induced by $Z$. Given $E^{\prime} \subseteq E$, we denote by weight $\left(E^{\prime}\right)$ the sum of weights of edges in $E^{\prime}$. Given a dual network we denote by $G_{p}[I], G_{c}[I]$, respectively, the subgraphs induced in the physical and conceptual network, respectively, by the set $I \subseteq V$.

A densest common subgraph $D C S$, formally defined in the following, is a subset of nodes $I$ that induces a connected subgraph in the conceptual network and a connected subgraph in the physical network.

Definition 3 Densest Common Subgraph.

Given a dual network $G\left(V, E_{c}, E_{p}\right)$, a densest common subgraph in $G\left(V, E_{c}, E_{p}\right)$ is a subset of nodes $I \subseteq V$ such that $G_{p}[I]$ is connected and the density of $G_{c}[I]$ is maximum.

In this paper, we are interested in finding $k \geq 1$ densest connected subgraphs. However, to avoid taking the same copy of a subgraph or subgraphs that are very similar, we consider the following distance functions introduced in Galbrun et al. (2016).

Definition 4 Let $G\left(V, E_{c}, E_{p}\right)$ be a dual network and let $G[A], G[B]$, with $A, B \subseteq V$, be two induced subgraphs of $G$. The distance between $G[A]$ and $G[B]$, denoted by $d: 2^{V} \times 2^{V} \rightarrow \mathbb{R}_{+}$has value equal $2-\frac{|A \cap B|^{2}}{|A||B|}$ if $A \neq B$, else is equal to 0 .

Notice that $2-\frac{|A \cap B|^{2}}{|A||B|}$ decreases as the overlapping between $A$ and $B$ increases.

Now, we are able to introduce the problem we are interested into.

Problem 1 Weighted-Top-k-Overlapping DCS

Input: A dual network $G\left(V, E_{c}, E_{p}\right)$, a parameter $\lambda>0$. 
Output: a set $\mathcal{X}=\left\{G\left[X_{1}\right], \ldots, G\left[X_{k}\right]\right\}$ of $k$ connected subgraphs of $G$, with $k \geq 1$, such that the following objective function is maximised:

$$
\sum_{i=1}^{k} \rho\left(G_{c}\left[X_{i}\right]\right)+\lambda \sum_{i=1}^{k-1} \sum_{j=i+1}^{k} d\left(G\left[X_{i}\right], G\left[X_{j}\right]\right)
$$

Weighted-Top-k-Overlapping DCS, for $k \geq 3$, is NP-hard, as it is NP-hard already on an unweighted graphs (Dondi et al. 2019). Notice that for $k=1$, then Weighted-Top-kOverlapping DCS is exactly the problem of finding a single weighted densest connected subgraph, hence it can be solved in polynomial time (Goldberg 1984).

\section{Greedy algorithms for DCS}

One of the ingredient of our method is a variant of a greedy algorithm for DCS, denoted by Greedy, which is an approximation algorithm for the problem of computing a connected densest subgraph of a given graph. Given a weighted graph $G$, Greedy (Asahiro et al. 2000; Charikar 2000) iteratively removes from $G$ a vertex $v$ having lowest vol $(v)$ and stops when all the vertices of the graph have been removed. It follows that at each iteration $i$, with $1 \leq i \leq|V|$, Greedy computes a subgraph $G_{i}$ of $G$. The output of this algorithm is a densest of subgraphs $G_{1}, \ldots, G_{|V|}$. The algorithm has a time complexity $O(|E|+|V| \log |V|)$ on weighted graphs and achieves an approximation factor of $\frac{1}{2}$ (Asahiro et al. 2000; Charikar 2000).

We introduce here a variant of the Greedy algorithm, called V-Greedy. Given an input weighted graph $G$, V-Greedy, similarly to Greedy, at each iteration $i$, with $1 \leq i \leq|V|$, removes a vertex $v$ having lowest $\operatorname{vol}(v)$ and computes a subgraph $G_{i}$, with $1 \leq i \leq|V|$. Then, among subgraphs $G_{1}, \ldots, G_{|V|}$, V-Greedy returns a subgraph $G_{i}$ that maximises the value:

$$
\rho\left(G_{i}\right)+2\left(\frac{\rho\left(G_{i}\right)}{\left|V_{i}\right|}\right) .
$$

Essentially, when selecting the subgraph to return among $G_{1}, \ldots, G_{|V|}$, we add to the density the correction factor $2\left(\frac{\rho\left(G_{i}\right)}{\left|V_{i}\right|}\right)$. This factor is added to avoid returning a subgraph that is not well-connected in terms of edge connectivity, that is it contains a small cut. For example, consider a graph with two equal size cliques $K_{1}$ and $K_{2}$ having the same (large) weighted density and a single edge of large weight connecting them. Then the union of $K_{1}$ and $K_{2}$ is denser than both $K_{1}$ and $K_{2}$, hence Greedy returns the union of $K_{1}$ and $K_{2}$. This may prevent us to find $K_{1}, K_{2}$ as a solution of Weighted-Top-k-Overlapping DCS. In this example, when the density of $K_{1}$ and $K_{2}$ is close enough to the density of their union, V-Greedy will return one of $K_{1}, K_{2}$.

\section{The proposed algorithm}

In this section we present our heuristic for Weighted-Top-k-Overlapping DCS in dual networks. The approach is based on two main steps:

1. First, the input networks are integrated into a single weighted alignment graph preserving the connectivity properties of the physical network 
2. Second, the obtained alignment graph is mined by using an ad-hoc heuristic for Weighted-Top-k-Overlapping DCS based on the V-Greedy algorithm

\section{Building of the alignment graph}

In the first step the algorithm receives in input: a weighted graph $G_{c}\left(V, E_{c}\right)$ (the conceptual graph); an unweighted graph $G_{p}\left(V, E_{p}\right)$ (the physical graph); an initial set (seed nodes) of node pairs $P$, where each pair defines a correspondence between a node of $G_{c}$ and a node of $G_{p}$; a distance threshold $\delta$ that represents the maximum threshold distance that two nodes may have in the physical network. For example, when $\delta$ is set to one, only adjacent nodes in both networks are considered.

Given the input data, the algorithm starts by building the nodes of the alignment graph. The alignment graph contains a node for each pair in $P$. The edges and weights of the alignment graph are defined as follows:

- An edge $\{u, v\}$ is defined in the alignment graph when the nodes corresponding to $u$ and $v$ are adjacent in $G_{p}$ and in $G_{c}$; the weight of $\{u, v\}$ is equal to the weight of the edge connecting the nodes corresponding to $u$ and $v$ in $G_{c}$

- An edge $\{u, v\}$ is defined in the alignment graph when $u$ and $v$ are adjacent in $G_{p}$ and have distance lower than $\delta$ in $G_{c}$; the weight of $\{u, v\}$ is equal to the average of the weights on a shortest path connecting the nodes corresponding to $u$ and $v$ in $G_{c}$.

\section{A heuristic for Weighted-top-k-overlapping DCS}

In the second phase of our algorithm, we solve Weighted-Top-k-Overlapping DCS on the alignment graph $G$ computed in phase 1 via a heuristic. We present here our heuristic for Weighted-Top-k-Overlapping DCS, called Iterative Weighted Dense Subgraphs (IWDS).

The heuristic starts with a set $\mathcal{X}=\emptyset$ and consists of $k$ iterations. At each iteration $i$, with $1 \leq i \leq k$, given a set $\mathcal{X}=\left\{G\left[X_{1}\right], \ldots, G\left[X_{i-1}\right]\right\}$ of subgraphs of $G$, IWDS computes a subgraph $G\left[X_{i}\right]$ and adds it to $\mathcal{X}$.

The first iteration of IWDS applies the V-Greedy algorithm (see "Greedy algorithms for DCS" section) on $G$ and computes $G\left[X_{1}\right]$. In iteration $i$, with $2 \leq i \leq k$, IWDS applies one of the two following cases, depending on a parameter $f, 0<f \leq 1$, and on the size of the set $C_{i-1}=\bigcup_{j=1}^{i-1} X_{j}$ (the set of nodes already covered by the subgraphs in $\mathcal{X}$ ).

Case 1. If $\left|C_{i-1}\right| \leq f|V|$ (that is at most $f|V|$ nodes of $G$ are covered by the subgraphs in $\mathcal{X}$ ), IWDS applies the V-Greedy algorithm on a subgraph $G^{\prime}$ pf $G$ obtained by retaining $\alpha$ nodes ( $\alpha$ is a parameter) of $C_{i-1}$ having highest weighted degree in $G$ and removing the other nodes of $C_{i-1} . G^{\prime}\left[X_{i}\right]$ is a weighted connected dense subgraph in $G^{\prime}$, distinct from those in $\mathcal{X}$.

Case 2. If $\left|C_{i-1}\right|>f|V|$ (more than $f|V|$ nodes of $G$ are covered by the subgraphs in $\mathcal{X})$, IWDS applies the V-Greedy algorithm on a subgraph $G^{\prime \prime}$ of $G$ obtained by removing $(1-\alpha)$ nodes (recall that $\alpha$ is a parameter of IWDS) of $C_{i-1}$ having lowest weighted degree in $G$. IWDS computes $G^{\prime \prime}\left[X_{i}\right]$ as a weighted connected dense subgraph in $G^{\prime}$, distinct from those in $\mathcal{X}$. 


\section{Complexity evaluation.}

We denote by $n$ (by $m$, respectively) the number of nodes (of edges, respectively) of the dual network. The first step requires the analysis of both the physical and the conceptual graph, and the construction of the novel alignment graph. This requires $\mathcal{O}\left(n^{2}\right)$ (calculation-edge-weights) time. The calculation of edge weights requires the calculation of a shortest path among all the node pairs in the physical graph using the Chan implementation (Chan 2012), therefore it requires $\mathcal{O}\left(n m_{p}\right)$ time $\left(m_{p}\right.$ is the number of edges of the physical graph).

As for Step 2, IWDS makes $k$ iterations. Each iteration applies V-Greedy on $G$ and requires $O(m n \log n)$ time, as the Greedy algorithm (Charikar 2000). Iteration $i$, with $2 \leq i \leq k$, first computes the set of covered nodes in order to find those nodes that have to be removed (or retained). For this purpose, we sort the nodes in $C_{j-1}$ based on their weighted degree in $O(n \log n)$ time. Thus the overall time complexity of IWDS is $O(k m n \log n)$.

\section{Experiments}

In this section, we provide an experimental evaluation of IWDS on synthetic and real networks. ${ }^{1}$ The design of a strong evaluation scheme for our algorithm is not simple, since we have to face two main issues:

1. Existing methods for computing the top $k$ overlapping subgraphs (Galbrun et al. 2016) are defined for unweighted graphs and cannot be used on dual networks.

2. Existing network alignment algorithms do not aim to extract top $k$ densest subgraphs.

Consequently, we cannot easily compare our approach with the existing state of the art methods, and we design an ad-hoc procedure for the evaluation of our method based on the following steps. First, we consider the performance of our approach on synthetic networks. In this way, we show that, in many of the cases we considered, IWDS can correctly recover top $k$ weighted densest subgraphs. Then we apply our method to four realworld dual networks.

The alignment algorithm described of "A heuristic for Weighted-top-k-overlapping DCS" section is implemented in Python 3.7 using the NetworkX package for managing networks (Hagberg et al. 2008). IWDS is implemented in MATLAB R2020a. We perform the experiments on MacBook-Pro (OS version 10.15.3) with processor $2.9 \mathrm{GHz}$ Intel Core i5 and 8 GB 2133 MHz LPDDR3 of RAM, Intel Iris Graphics 5501536 MB.

\section{Synthetic networks}

In the first part of our experimental evaluation, we analyse the performance of IWDS to find planted ground-truth subgraphs on synthetic datasets.

\footnotetext{
$\overline{1}$ The source code and data used in our experiments are available at https://github.com/mehdihosseinzadeh/-k-overl apping-densest-connected-subgraphs.
} 
Datasets. We generate two noiseless synthetic datasets, consisting of $k=5$ planted dense subgraphs (cliques). Synthetic1 contains five non-overlapping ground-truth subgraphs, while Synthetic3 contains five overlapping ground-truth subgraphs.

In Synthetic1, each planted dense subgraph contains 30 nodes and has edge weights randomly generated in the interval [0.8, 1]. In Synthetic3, each planted dense subgraph contains 20 nodes not shared with other planted subgraphs. The subgraphs are arranged in a cycle, 5 nodes of each subgraph are shared with the subgraph on one side and 5 nodes are shared with the subgraph on the other side. Edge weights are randomly generated in the interval $[0.8,1]$.

These cliques are then connected to a background subgraph of 100 nodes. We consider three different ways to generate the background subgraph: Erdös-Renyi with parameter $p=0.1$, Erdös-Renyi with parameter $p=0.2$ and Barabasi-Albert with parameter equal to 10 . Weights of the background graphs are randomly generated in interval $[0,0.5]$. Then 50 edges connecting cliques and the background graph are randomly added (with weights randomly generated in interval $[0,0.5]$ ).

Based on this approach, we generate four different sets of synthetic networks, called Synthetic1, Synthetic2, Synthetic3 and Synthetic4. Synthetic1 (for the non-overlapping case) and Synthetic3 (for the overlapping case) are generated as described above. Synthetic2 and Synthetic4, respectively, are obtained by applying noise to the synthetic networks in Synthetic1, Synthetic3, respectively. The noise is added by varying 5\%,10\% and $15 \%$ of node relations of each network. A set of pairs of nodes are chosen randomly: if they belong to the same clique, the weight of the edge connecting the two nodes is changed to a random value in the interval $[0,0.5]$; else an edge connecting the two nodes is (possibly) added (if not already in the network) and its weight is randomly assigned a value in the interval $[0.8,1]$.

Outcome. We present the results of our experimental evaluation, in particular, the average running time, density, distance and F1-score, ${ }^{2}$ varying the parameter $\alpha$. We recall that F1-score is the average mean of precision and recall, and, as in Galbrun et al. (2016) we consider this measure to evaluate the accuracy of our method to detect the ground-truth subgraphs. Following Yang and Leskovec (2012), we consider the number of shared nodes between each ground-truth subgraph and each detected subgraph, so that we are able to define the best-matching of ground-truth subgraphs and detected subgraphs. Then, we compute the $F 1[t / d]$ measure as the average F1-score of the bestmatching ground-truth subgraph to each detected subgraph (truth to detected) and $F 1[d / t]$ measure as the average F1-score of the best-matching detected subgraph to each ground-truth subgraph (detected to truth). Notice that in most of the cases considered, the running time of IWDS increases with the increasing of $\alpha$. Also, generally, the solutions returned by IWDS for larger values of $\alpha$ are denser than for small values, while the solutions with small values of $\alpha$ have a higher value of distance (hence the subgraphs returned have a smaller overlapping).

\footnotetext{
${ }^{2}$ Given the ground-truth and detected subgraphs, F1-score is calculated considering precision and recall, where precision is the fraction of the number of nodes in the ground-truth correctly identified by detected subgraphs divided by number of nodes in the detected subgraphs, whereas the recall is the fraction of the number nodes in the ground-truth correctly identified by the detected subgraphs divided by the number of nodes in the ground-truth.
} 
Table 1 Performance of IWDS on non overlapping generated networks (called synthetic 1 ) for $k=5$, varying $\alpha$ from 0.05 to 0.9 , the running time (in minutes), the density and the distance are averaged over 300 examples

\begin{tabular}{lllllll}
\hline & $\alpha=0.05$ & $\alpha=0.1$ & $\alpha=0.25$ & $\alpha=0.5$ & $\alpha=0.75$ & $\alpha=0.9$ \\
\hline Time & 0.0188 & 0.0187 & 0.0194 & 0.0217 & 0.0259 & 0.0231 \\
Density & 65.28 & 65.28 & 65.28 & 65.28 & 65.28 & 65.28 \\
Distance & 20 & 20 & 20 & 20 & 20 & 20 \\
$F 1[t / d]$ & 1.00 & 1.00 & 1.00 & 1.00 & 1.00 & 1.00 \\
$F 1[d / t]$ & 1.00 & 1.00 & 1.00 & 1.00 & 1.00 & 1.00 \\
\hline
\end{tabular}

Table 2 Performance of IWDS on overlapping generated networks (called synthetic3) for $k=5$, varying $\alpha$ from 0.05 to 0.9 , the running time (in minutes), the density and the distance are averaged over 300 examples

\begin{tabular}{lllllll}
\hline & $\alpha=0.05$ & $\alpha=0.1$ & $\alpha=0.25$ & $\alpha=0.5$ & $\alpha=0.75$ & $\alpha=0.9$ \\
\hline Time & 0.0104 & 0.0128 & 0.0145 & 0.0170 & 0.0188 & 0.0209 \\
Density & 21.00 & 23.41 & 32.29 & 46.11 & 57.95 & 64.22 \\
Distance & 18.178 & 17.473 & 16.321 & 15.853 & 15.741 & 15.344 \\
$F 1[t / d]$ & 0.509 & 0.415 & 0.689 & 0.768 & 0.745 & 0.456 \\
$F 1[d / t]$ & 0.101 & 0.157 & 0.331 & 0.583 & 0.804 & 0.923 \\
\hline
\end{tabular}

Tables 1 and 3 report average results of running time (in minutes), density, distance and F1 scores for the two noiseless datasets. Table 1 shows the experimental results for the noiseless Synthetic1 dataset, where ground-truth subgraphs are disjoint. In this case IWDS is able to detect the ground-truth subgraphs for all values of $\alpha$, averaged over 300 examples. Table 2 shows the experimental results for the noiseless Synthetic3 dataset, where ground-truth subgraphs are overlapping. In this case the best performances are achieved for $\alpha=0.75$, where F1[t/d] $=0.745$, while $\mathrm{F} 1[\mathrm{~d} / \mathrm{t}]=0.804$. The experimental results show that $\mathrm{F} 1[\mathrm{~d} / \mathrm{t}]$ increases with $\alpha$, in particular for lower values of $\alpha(\alpha \leq 0.25)$ the performance of IWDS for this measure is poor. We observe that for values of $\alpha \geq 0.5$, the F1[t/d] measure decreases as $\alpha$ increases.

Tables 3 and 4 show the performances of IWDS on the noisy datasets Synthetic2 and Synthetic4. Recall that for these datasets, we consider noise values of 0.05, 0.10 and 0.15 . The results we present are averaged over 90 examples. As for the noiseless datasets, we vary the value of parameter $\alpha$.

For Synthetic2, for noise value 0.05 and 0.10 , we obtain near optimal solutions for all the cases considered. The performances of IWDS starts to degrade with noise equal to 0.15 , in particular the values of F1[d/t] for $\alpha \leq 0.25$. F1 $[\mathrm{t} / \mathrm{d}]$ is instead close to 1 (at least 0.93 ) for the values of $\alpha$ considered.

For Synthetic4, the added noise has a significant impact on the quality of computed solutions, even for noise value equal to 0.05 . While the noise increasing has a limited effect on IWDS for small value of $\alpha(\alpha \leq 0.25)$, for higher values of $\alpha$ leads to a degrade in performance, in particular for $\mathrm{F} 1[\mathrm{t} / \mathrm{d}]$. 
Table 3 Performance of IWDS on non overlapping generated networks with added noise varying from 0.05 to 0.15 (called synthetic2) for $k=5$, varying $\alpha$ from 0.05 to 0.9 , the running time (in minutes), the density and the distance are averaged over 90 examples

\begin{tabular}{|c|c|c|c|c|c|c|}
\hline Noise & $\alpha=0.05$ & $\alpha=0.1$ & $\alpha=0.25$ & $\alpha=0.5$ & $\alpha=0.75$ & $\alpha=0.9$ \\
\hline \multicolumn{7}{|l|}{0.05} \\
\hline Time & 0.0181 & 0.0181 & 0.0203 & 0.0214 & 0.0222 & 0.0236 \\
\hline Density & 65.46 & 65.46 & 65.48 & 65.53 & 65.55 & 65.55 \\
\hline Distance & 20 & 19.998 & 19.996 & 19.996 & 19.991 & 19.850 \\
\hline$F 1[t / d]$ & 0.989 & 1.00 & 1.00 & 1.00 & 1.00 & 1.00 \\
\hline$F 1[d / t]$ & 0.990 & 0.991 & 0.993 & 0.996 & 0.998 & 0.995 \\
\hline \multicolumn{7}{|l|}{0.10} \\
\hline Time & 0.0187 & 0.0179 & 0.0207 & 0.0199 & 0.0233 & 0.0238 \\
\hline Density & 65.42 & 65.42 & 65.53 & 65.72 & 65.89 & 66.00 \\
\hline Distance & 19.999 & 19.999 & 19.986 & 19.976 & 19.960 & 19.847 \\
\hline$F 1[t / d]$ & 0.978 & 0.968 & 1.00 & 1.00 & 1.00 & 1.00 \\
\hline$F 1[d / t]$ & 0.960 & 0.962 & 0.970 & 0.982 & 0.992 & 0.994 \\
\hline \multicolumn{7}{|l|}{0.15} \\
\hline Time & 0.0126 & 0.0131 & 0.0164 & 0.0194 & 0.0230 & 0.0241 \\
\hline Density & 36.63 & 39.35 & 43.03 & 51.57 & 59.67 & 64.47 \\
\hline Distance & 19.439 & 19.111 & 18.218 & 18.112 & 18.083 & 18.056 \\
\hline$F 1[t / d]$ & 0.93 & 0.95 & 0.93 & 0.98 & 0.95 & 0.94 \\
\hline$F 1[d / t]$ & 0.41 & 0.47 & 0.54 & 0.70 & 0.85 & 0.94 \\
\hline
\end{tabular}

Table 4 Performance of IWDS on overlapping generated networks with added noise varying from 0.05 to 0.15 (called synthetic4) for $k=5$, varying $\alpha$ from 0.05 to 0.9 , the running time (in minutes), the density and the distance are averaged over 90 examples

\begin{tabular}{|c|c|c|c|c|c|c|}
\hline Noise & $\alpha=0.05$ & $\alpha=0.1$ & $\alpha=0.25$ & $\alpha=0.5$ & $\alpha=0.75$ & $\alpha=0.9$ \\
\hline \multicolumn{7}{|l|}{0.05} \\
\hline Time & 0.0090 & 0.0112 & 0.0149 & 0.0180 & 0.0203 & 0.0205 \\
\hline Density & 21.34 & 24.89 & 32.09 & 45.92 & 57.31 & 63.50 \\
\hline Distance & 18.361 & 17.506 & 15.823 & 15.550 & 15.220 & 15.024 \\
\hline$F 1[t / d]$ & 0.649 & 0.660 & 0.563 & 0.692 & 0.631 & 0.527 \\
\hline$F 1[d / t]$ & 0.131 & 0.228 & 0.332 & 0.589 & 0.806 & 0.927 \\
\hline \multicolumn{7}{|l|}{0.10} \\
\hline Time & 0.0098 & 0.0118 & 0.0137 & 0.0178 & 0.0195 & 0.0212 \\
\hline Density & 21.95 & 25.54 & 32.72 & 46.35 & 58.43 & 65.25 \\
\hline Distance & 18.275 & 17.260 & 15.761 & 15.229 & 14.876 & 13.847 \\
\hline$F 1[t / d]$ & 0.648 & 0.568 & 0.567 & 0.595 & 0.548 & 0.463 \\
\hline$F 1[d / t]$ & 0.131 & 0.225 & 0.330 & 0.581 & 0.807 & 0.936 \\
\hline \multicolumn{7}{|l|}{0.15} \\
\hline Time & 0.0092 & 0.0113 & 0.0149 & 0.0178 & 0.02 & 0.0218 \\
\hline Density & 22.40 & 26.06 & 32.98 & 46.68 & 58.91 & 65.75 \\
\hline Distance & 18.213 & 17.189 & 15.332 & 14.932 & 14.263 & 12.717 \\
\hline$F 1[t / d]$ & 0.624 & 0.555 & 0.501 & 0.539 & 0.419 & 0.303 \\
\hline$F 1[d / t]$ & 0.134 & 0.223 & 0.336 & 0.586 & 0.811 & 0.938 \\
\hline
\end{tabular}


Table 5 Properties of the alignment graphs obtained for each dataset

\begin{tabular}{llll}
\hline Graph & Represented relation & Nodes & Edges \\
\hline DBLP-graphA & Co-authorship & 18,954 & 553,699 \\
G-graphA & Social & 9878 & $2,241,339$ \\
HS-graphA & Protein interactions & 19,354 & $5,879,727$ \\
Protein-interaction & Protein interactions & 192 & 418 \\
\hline
\end{tabular}

\section{Dual networks}

We evaluate IWDS on four real-world dual network datasets:

Datasets. G-graphA. The G-graphA dataset is derived from the GoWalla social network where users share their locations (expressed as GPS coordinates) by checking-in into the web site (Cho et al. 2011). Each node represents a user and each edge links two friends in the network. We obtained the physical network by considering friendship relation on the social network. We calculated the conceptual network by considering the distance among users. Then we run the first step of our algorithm and we obtained the alignment graph G-graphA, containing 2,241,339 interactions and 9878 nodes (we set $\delta$ $=4)$. In this case a DCS represents set of friends that share check-ins in near locations.

$D B L P$-graphA. The DBLP-graphA dataset is extracted from a computer science bibliography and represents interactions between authors. Nodes represent authors and edges represent connections between two authors if they have published at least one paper together. Each edge in the physical network connects two authors that coauthored at least one paper. Edges in the conceptual network represent the similarity of research interests of the authors calculated on the basis of all their publications. After running the first step of the algorithm (using $\delta=4$ ), we obtained an alignment graph DBLP-graphA dataset containing 553,699 interactions and 18,954 nodes. In this case a DCS represents a set of co-authors that share some strong common research interests and the use of DNs is mandatory, since physical network shows only co-authors that may not have many common interests and the conceptual network represents authors with common interest that may not be co-authors.

HS-graphA. HS-graphA is a biological dataset and is taken from the STRING database (Szklarczyk et al. 2016). Each node represents a protein, and each edge takes into account the reliability of the interactions. We use two networks for modelling the database: a conceptual network represents such reliability value; and a physical network stores the binary interactions. The $H S$-graphA dataset contains 5,879,727 interactions and 19,354 nodes (we set $\delta=4$ ).

Protein-interaction We extracted from the STRING database a subnetwork of proteins involved into the SARS-COV-2 infection (Szklarczyk et al. 2016). The physical network contains interacting proteins, while the conceptual network contains the strength of the association among them. Protein-Interaction contains 192 nodes and 418 edges (Table 5).

\section{Outcome}

For these large size datasets, we set the value of $k$ to 20, following the approach in Galbrun et al. (2016). Table 6 reports the running time of IWDS, and the density and distance of the solutions returned by IWDS. As for the synthetic datasets, we consider six 
Table 6 Performance of IWDS on real-world network for $k=20$, varying $\alpha$ from 0.05 to 0.9 . For each network, we report the running time in minutes, the density and the distance

\begin{tabular}{lllllll}
\hline Set & $\alpha=0.05$ & $\alpha=0.1$ & $\alpha=0.25$ & $\alpha=0.5$ & $\alpha=0.75$ & $\alpha=0.9$ \\
\hline \multicolumn{2}{l}{ Alignment-graph } & & & & & \\
Time & 0.055 & 0.058 & 0.062 & 0.065 & 0.068 & 0.068 \\
Density & 28.14 & 30.45 & 37.14 & 46.44 & 47.73 & 52.94 \\
Distance & 378.76 & 373.61 & 359.94 & 351.50 & 347.81 & 339.17 \\
G-graphA & & & & & & \\
Time & 89.84 & 98.72 & 184.87 & 336.72 & 426.56 & 486.68 \\
Density & 2863.99 & 4000.73 & 6345.67 & 10989.07 & 9297.13 & 10737.01 \\
Distance & 275.82 & 257.84 & 220.16 & 210.79 & 196.06 & 193.02 \\
DBLP-graphA & & & & & & \\
Time & 105.69 & 125.71 & 165.25 & 212.07 & 251.08 & 277.39 \\
Density & 39.61 & 52.39 & 74.12 & 91.13 & 97.25 & 98.78 \\
Distance & 307.72 & 231.25 & 213.04 & 204.37 & 198.54 & 196.96 \\
HS-graphA & & & & & & - \\
Time & 209.88 & 749.06 & 1027.58 & - & & \\
Density & 1326.07 & 1153.68 & 1799.22 & & & \\
Distance & 226.40 & 212.34 & 205.55 & & & \\
\hline
\end{tabular}

different values of $\alpha$. As shown in Table 6, by increasing the value of $\alpha$ from 0.05 to 0.5 , IWDS (except of one case, HS-graphA with $\alpha=0.1$ ) returns solutions that are denser, but with lower distance.

Table 6 shows also how the running time of IWDS is influenced by the size of the network and by the value of $\alpha$. We have put a bound of $20 \mathrm{~h}$ on the running time of IWDS and the method was not able to return a solution for HS-graphA for $\alpha \geq 0.5$ within this time. The running time is influenced in particular by the number of edges of the input network. DBLP-graphA and HS-graph-A have almost the same number of nodes, but HS-graph-A is much more denser than DBLP-graphA. IWDS for the former network is remarkably slower than for DBLP-graphA (1.986 slower for $\alpha=0.05,6.218$ slower for $\alpha=0.25)$. The running time of IWDS is considerably influenced by the value of parameter $\alpha$, since it increases as $\alpha$ increases. Indeed by increasing the value of $\alpha$, less nodes are removed by Case 1 and Case 2 of IWDS, hence in iterations of IWDS V-Greedy is applied to larger subgraphs. This fact can be seen in particular for HS-graphA, for which IWDS failed to terminate within $20 \mathrm{~h}$ when $\alpha \geq 0.5$.

\section{Biological evaluation of results}

For biological data there is the possibility to evaluate the relevance of the results considering the relevance of the biological knowledge that results may convey.

Biological data are usually annotated with terms extracted from ontologies, e.g. Gene Ontology (Guzzi et al. 2012). Consequently, experiments of analysis of biological data may evaluated in terms of the biological knowledge inferred from the analysis of data and in terms of the statistical relevance of the results themselves. For instance, given a DCS extracted from two biological networks, it is interesting to determine the biological meaning of the DCS and how this is relevant, i.e. how this DCS may convey biological relevance with respect to another random one. Usually, 
Table 7 Comparison of the average semantic similarity for the two biological networks considered

\begin{tabular}{ll}
\hline & Semantic similarity \\
\hline Random solutions & $0.3 \pm 0.1$ \\
DCS & $0.6 \pm 0.1$ \\
\hline
\end{tabular}

subgraphs of biological networks may represent groups of interacting proteins sharing some common functions or playing similar biological roles. Consequently, it is possible to evaluate the biological relevance of obtained results by considering the role of proteins. Such information are stored and organised into biological ontologies such as Gene Ontology (GO) (Harris et al. 2004). GO functional enrichment has been proposed to evaluate the significant presence of common roles or function in a solution represented as a list of genes/proteins. It has been shown that the use of semantic similarities (SS) (Guzzi et al. 2012) is a feasible and efficient way to quantify biological similarity among proteins. SS measures are able to quantify the functional similarity of pairs of proteins/genes, comparing the GO terms that annotate them, therefore proteins that share the biological role have high values of semantic similarity. As a consequence, genes/proteins that are found in the same solution should have a semantic similarity significantly higher than random expectation. These considerations have been used during the design of the evaluation of our results that we adapted from the evaluation scheme proposed in Mina and Guzzi (2014).

Given a DCS $D C S_{k}$ we calculate its internal semantic similarity $S S_{D C S_{k}}$ as the average semantic similarity of all the nodes pairs of the DCS as follows:

$$
S S_{D C S_{k}}=\frac{\sum_{n_{i} \in D C S_{k}} \sum_{n_{j} \in D C S_{k}, j \neq i} S_{D C S_{k}}\left(n_{i}, n_{j}\right)}{\left\|S S_{D C S_{k}}\right\|\left\|S S_{D C S_{k}-1}\right\|}
$$

We compare the DCS extracted from the biological network against random ones obtained by randomly sampling the input networks to prove their statistical significance. Given a DCS $D C S_{i}$, we can test the null hypothesis: $H_{1}^{0}$ : the average semantic similarity of the protein internals to the DCS $S S\left(D C S_{i}\right)$ is higher than by chance, where the background distribution can be estimated from the semantic similarity of random subgraphs $R S_{i}$ taken from the alignment graph $S S\left(R S_{i}\right)$, using for instance 0.05 as significance level.

Consequently we design this test as described in the following algorithm:

- Let $D C S_{i}$ be a given DCS;

- Let $S S\left(D C S_{i}\right)$ be its internal semantic similarity

- Let $V_{s}$ be the set of 100 random subgraph with same size $V_{s}=\left\{R S_{j}\right\} j=0, . ., 99$

- For Each $R S_{j} \in V_{s}$ calculate $S S_{j}\left(R S_{j}\right)$ the internal semantic similarity of each random solution

- Compare $S S\left(D C S_{i}\right)$ and all the $S S_{j}\left(R S_{j}\right)$ using a non parametric test

- Accept or Refuse the Hypothesis $S S\left(D C S_{i}\right)$ is significantly higher than $S S_{j}\left(R S_{j}\right)$

Consequently, for each graph in the solution we generate 100 random graphs of the same size, by sampling the obtained alignment graph. For each graph we calculated its internal semantic similarity using the Resnick measure (Resnik 1999). Results demonstrate that 
our solution is biologically relevant and the relevance is higher than by chance as summarised in Table 7.

\section{Conclusion}

DNs are used to model two kinds of relationships among elements in the same scenario. A DN is a pair of networks that have the same set of nodes. One network has unweighted edges (physical network), while the second one has weighted edges (conceptual network). In this contribution, we introduced an approach that first integrates a physical and a conceptual network into an alignment graph. Then, we applied the WeightedTop-k-Overlapping DCS problem to the alignment graph to find $k$ dense connected subgraphs. These subgraphs represent subsets of nodes that are strongly related in the conceptual network and that are connected in the physical one. We presented a heuristic, called IWDS, for Weighted-Top-k-Overlapping DCS and an experimental evaluation of IWDS. We first considered as a proof-of-concept the ability of our algorithm to retrieve known densest subgraphs in synthetic networks. Then we tested the approach on four real networks to demonstrate the effectiveness of our approach. Future work will consider a possible high performance implementation of our approach and the application of the IWDS algorithm to other scenarios (e.g. financial or marketing datasets).

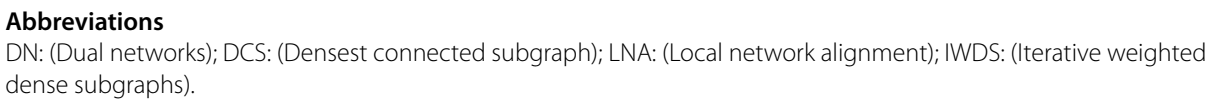

Acknowledgements

A preliminary version of the paper has been published in Dondi et al. (2020)

\section{Authors' contributions}

All the authors contribute to the framework definition. PHG designed and implemented the graph alignment part. RD and $\mathrm{MH}$ designed and implemented the heuristic IWDS. All the authors performed the experimental analysis. All the authors contributed to the manuscript writing. All the authors read and approved the manuscript.

Availability of data and materials

https://github.com/mehdihosseinzadeh/-k-overlapping-densest-connected-subgraphs

\section{Declarations}

Competing interests

The authors declare that they have no competing interests.

\section{Consent for publication}

We give our consent for the publication.

Author details

${ }^{1}$ Department of Science, University of Bergamo, Bergamo, Italy. ${ }^{2}$ Department of Surgical and Medical Sciences, Magna Graecia University, Catanzaro, Italy.

Received: 26 February 2021 Accepted: 20 May 2021

Published online: 05 June 2021

\section{References}

Abatangelo L, Maglietta R, Distaso A, D'Addabbo A, Creanza TM, Mukherjee S, Ancona N (2009) Comparative study of gene set enrichment methods. BMC Bioinform 10:275. https://doi.org/10.1186/1471-2105-10-275

Asahiro Y, Iwama K, Tamaki H, Tokuyama T (2000) Greedily finding a dense subgraph. J Algorithms 34(2):203-221

Balalau OD, Bonchi F, Chan T-H, Gullo F, Sozio M (2015) Finding subgraphs with maximum total density and limited overlap. In: Cheng, X., Li, H., Gabrilovich, E., Tang, J. (eds.) Proceedings of the eighth ACM international conference on web search and data mining, WSDM 2015, Shanghai, China, February 2-6, 2015. ACM, pp 379-388. https://doi.org/ $10.1145 / 2684822.2685298$ 
Barabási A-L (2011) The network takeover. Nat Phys 8(1):14-16. https://doi.org/10.1038/nphys2188

Cannataro M, Guzzi PH, Veltri P (2010) Protein-to-protein interactions. ACM Comput Surv 43(1):1-36. https://doi.org/10. 1145/1824795.1824796

Cannataro M, Guzzi PH, Veltri P (2010) Impreco: distributed prediction of protein complexes. Future Gener Comput Syst 26(3):434-440

Chan TM (2012) All-pairs shortest paths for unweighted undirected graphs in o(mn) time. ACM Trans Algorithms 8(4)

Charikar M (2000) Greedy approximation algorithms for finding dense components in a graph. In: International workshop on approximation algorithms for combinatorial optimization. Springer, pp 84-95

Charikar M (2000) Greedy approximation algorithms for finding dense components in a graph. In: Jansen K, Khuller S (eds) Approximation algorithms for combinatorial optimization, third international workshop, APPROX 2000, Proceedings. Lecture notes in computer science, vol 1913. Springer, pp 84-95. https://doi.org/10.1007/3-540-44436-X

Cho Y-R, Mina M, Lu Y, Kwon N, Guzzi PH (2013) M-finder: uncovering functionally associated proteins from interactome data integrated with go annotations. Proteome Sci 11(1):1-12

Cho E, Myers SA, Leskovec J (2011) Friendship and mobility: user movement in location-based social networks. In: Proceedings of the 17th ACM SIGKDD international conference on knowledge discovery and data mining. ACM, pp 1082-1090

Ciriello G, Mina M, Guzzi PH, Cannataro M, Guerra C (2012) AlignNemo: a local network alignment method to integrate homology and topology. PLOS ONE 7(6):38107. https://doi.org/10.1371/journal.pone.0038107

Clark C, Kalita J (2014) A comparison of algorithms for the pairwise alignment of biological networks. Bioinformatics (Oxford, England) 30(16):2351-2359

Dondi R, Mauri G, Sikora F, Zoppis I (2019) Covering a graph with clubs. J Graph Algorithms Appl 23(2):271-292. https:// doi.org/10.7155/jgaa.00491

Dondi R, Guzzi PH, Hosseinzadeh MM (2020) Top-k connected overlapping densest subgraphs in dual networks. In: International conference on complex networks and their applications. Springer, pp 585-596

Dondi R, Hosseinzadeh MM, Mauri G, Zoppis I (2019) Top-k overlapping densest subgraphs: approximation and complexity. In: Proceedings of the 20th Italian conference on theoretical computer science, ICTCS 2019, Como, Italy, September 9-11, 2019, pp 110-121

Faisal F, Meng L, Crawford J, Milenkovic T (2015) The post-genomic era of biological network alignment. EURASIP J Bioinform Syst Biol 2015(1):1-19

Galbrun E, Gionis A, Tatti N (2016) Top-k overlapping densest subgraphs. Data Min Knowl Discov 30(5):1134-1165. https://doi.org/10.1007/s10618-016-0464-z

Goldberg A (1984) Finding a maximum density subgraph. Technical report. University of California, Berkeley

Guzzi PH, Milenković T (2017) Survey of local and global biological network alignment: the need to reconcile the two sides of the same coin. Brief Bioinform 132

Guzzi PH, Cannataro M (2010) $\mu$-cs: an extension of the tm4 platform to manage affymetrix binary data. BMC Bioinform 11(1):315

Guzzi P, Mina M, Guerra C, Cannataro M (2012) Semantic similarity analysis of protein data: assessment with biological features and issues. Brief Bioinform 13(5):569-585. https://doi.org/10.1093/bib/bbr066

Guzzi PH, Salerno E, Tradigo G, Veltri P (2020) Extracting dense and connected communities in dual networks: an alignment based algorithm. IEEE Access 8:162279-162289

Hagberg A, Swart P, S Chult D (2008) Exploring network structure, dynamics, and function using network. In: Technical report, Los Alamos National Lab. (LANL), Los Alamos

Harris MA, Clark J, Ireland A, Lomax J, Ashburner M et al (2004) The gene ontology (go) database and informatics resource. Nucl Acids Res 32(Database issue):258-261

Hastad J (1996) Clique is hard to approximate within n/sup 1-/spl epsiv. In: Proceedings of 37th conference on foundations of computer science. IEEE, pp 627-636

Hosseinzadeh MM (2020) Dense subgraphs in biological networks. In: International conference on current trends in theory and practice of informatics. Springer, pp 711-719

Karp RM (2009) Reducibility among combinatorial problems. In: 50 years of integer programming 1958-2008. Springer, Berlin, pp 219-241

Kawase Y, Miyauchi A (2018) The densest subgraph problem with a convex/concave size function. Algorithmica 80(12):3461-3480. https://doi.org/10.1007/s00453-017-0400-7

Komusiewicz C (2016) Multivariate algorithmics for finding cohesive subnetworks. Algorithms 9(1):21

Liu X, Shen C, Guan X, Zhou Y (2018) Digger: detect similar groups in heterogeneous social networks. ACM Trans Knowl Discov from Data (TKDD) 13(1):2

Milano M, Guzzi PH, Cannataro M (2018) Glalign: a novel algorithm for local network alignment. IEEE/ACM Trans Comput Biol Bioinform 16(6):1958-1969

Milano M, Milenković T, Cannataro M, Guzzi PH (2020) L-HetNetAligner: a novel algorithm for local alignment of heterogeneous biological networks. Sci Rep 10(1):3901. https://doi.org/10.1038/s41598-020-60737-5

Mina M, Guzzi PH (2014) Improving the robustness of local network alignment: design and extensive assessment of a Markov clustering-based approach. IEEE/ACM Trans Comput Biol Bioinform (TCBB) 11(3):561-572

Phillips PC (2008) Epistasis - the essential role of gene interactions in the structure and evolution of genetic systems. Nat Rev Genet 9(11):855-867

Resnik P (1999) Semantic similarity in a taxonomy: an information-based measure and its application to problems of ambiguity in natural language. J Artif Intell Res 11:95-130

Sapountzi A, Psannis KE (2018) Social networking data analysis tools and challenges. Future Gener Comput Syst 86:893-913

Sun YV, Kardia SL (2010) Identification of epistatic effects using a protein-protein interaction database. Human Mol Genet 19(22):4345-4352 
Szklarczyk D, Morris JH, Cook H, Kuhn M, Wyder S, Simonovic M, Santos A, Doncheva NT, Roth A, Bork P et al (2016) The string database in 2017: quality-controlled protein-protein association networks, made broadly accessible. Nucl Acids Res 937

Wu Y, Zhu X, Li L, Fan W, Jin R, Zhang X (2016) Mining dual networks: models, algorithms, and applications. TKDD

Yang J, Leskovec J (2012) Community-affiliation graph model for overlapping network community detection. In: 2012 IEEE 12th international conference on data mining. IEEE, pp 1170-1175

Zuckerman D (2006) Linear degree extractors and the inapproximability of max clique and chromatic number. In: Kleinberg JM (ed) Proceedings of the 38th annual ACM symposium on theory of computing, Seattle, WA, USA, May 21-23, 2006. ACM, pp 681-690 (2006). https://doi.org/10.1145/1132516.1132612

\section{Publisher's Note}

Springer Nature remains neutral with regard to jurisdictional claims in published maps and institutional affiliations.

\section{Submit your manuscript to a SpringerOpen ${ }^{\circ}$} journal and benefit from:

- Convenient online submission

- Rigorous peer review

- Open access: articles freely available online

- High visibility within the field

- Retaining the copyright to your article

Submit your next manuscript at $\boldsymbol{\Delta}$ springeropen.com 